\title{
Fuzzy Number - A New Hypothesis and Solution of Fuzzy Equations
}

\author{
Vijay C. Makwana ${ }^{1}$, Vijay P. Soni ${ }^{1}$, Nayan I. Patel ${ }^{2}$, Manoj Sahni ${ }^{3, *}$ \\ ${ }^{1}$ Department of Mathematics, Government Engineering College, Patan, Gujarat, India \\ ${ }^{2}$ Department of Mathematics, Adani Institute of Infrastructure Engineering, Ahmedabad, Gujarat, India \\ ${ }^{3}$ Department of Mathematics, Pandit Deendayal Energy University, Gandhinagar, Gujarat, India
}

Received November 16, 2021; Revised December 17, 2021; Accepted January 25, 2022

\section{Cite This Paper in the following Citation Styles}

(a): [1] Vijay C. Makwana, Vijay P. Soni, Nayan I. Patel, Manoj Sahni, "Fuzzy Number - A New Hypothesis and Solution of Fuzzy Equations," Mathematics and Statistics, Vol. 10, No. 1, pp. 176 - 186, 2022. DOI: 10.13189/ms.2022.100116.

(b): Vijay C. Makwana, Vijay P. Soni, Nayan I. Patel, Manoj Sahni (2022). Fuzzy Number - A New Hypothesis and Solution of Fuzzy Equations. Mathematics and Statistics, 10(1), 176 - 186. DOI: 10.13189/ms.2022.100116.

Copyright $\mathrm{C} 2022$ by authors, all rights reserved. Authors agree that this article remains permanently open access under the terms of the Creative Commons Attribution License 4.0 International License

\begin{abstract}
In this paper, a new hypothesis of fuzzy number has been proposed which is more precise and direct. This new proposed approach is considered as an equivalence class on set of real numbers $R$ with its algebraic structure and its properties along with theoretical study and computational results. Newly defined hypothesis provides a well-structured summary that offers both a deeper knowledge about the theory of fuzzy numbers and an extensive view on its algebra. We defined field of newly defined fuzzy numbers which opens new era in future for fuzzy mathematics. It is shown that, by using newly defined fuzzy number and its membership function, we are able to solve fuzzy equations in an uncertain environment. We have illustrated solution of fuzzy linear and quadratic equations using the defined new fuzzy number. This can be extended to higher order polynomial equations in future. The linear fuzzy equations have numerous applications in science and engineering. We may develop some iterative methods for system of fuzzy linear equations in a very simple and ordinary way by using this new methodology. This is an innovative and purposefulness study of fuzzy numbers along with replacement of this newly defined fuzzy number with ordinary fuzzy number.
\end{abstract}

Keywords Fuzzy Number, Arithmetic of Fuzzy Numbers, Fuzzy Algebraic Structure, Solution of Fuzzy Equation

\section{Introduction}

In the current scenario, many real life problems cannot be dealt with the classical set theory and hence a need arises of its extension. So, in 1960's an eminent scientist Zadeh [1] proposed a novel theory known as Fuzzy Sets, a generalization of classical set theory. This fuzzy set theory deals with vagueness which the classical set theory was unable to do so. In 1986, Atanassov [2] has proposed Intuitionistic Fuzzy Sets and shown that it is a generalization of fuzzy sets. In continuation, lot of extensions covering various applications are being developed and some of them are like ortho-pair, neutrosophic, etc. The concept of fuzzy number [3] was introduced by Zadeh. The literature review $[3,5]$ done by us shows that different operations were defined on fuzzy numbers and their applications are shown. The use of fuzzy numbers is used in solving fuzzy equations [4, 7-10], fuzzy calculus [11-12], fuzzy graph [13], fuzzy differential equations [14-15], and many other areas of pure and applied sciences. Esa et al. [16] has solved Fuzzy Volterra Integral Equation by Sixth Order Runge-Kutta numerical method. The study of modification of fuzzy numbers may useful to understand the difference between oriented fuzzy numbers and Fuzzy numbers [5]. This study may be considered as an extension of triangular and diamond fuzzy number [6]. The canonical representation of fuzzy number was given by Delgado et al. in 1998 [17].

Solving fuzzy equations has long been a problem in fuzzy set theory. The classical methods, involving the extension principle and alpha-cuts are too restrictive because very often there is no solution or very strong conditions must be 
placed on equations so that there will be a solution. This made it very difficult to apply fuzzy set theory to algebra. These facts motivated us to develop a new solution technique which may be suitable for a given problem.

In this paper, we introduced new hypothesis of fuzzy numbers and their fuzzy arithmetic operations on new fuzzy numbers. The flow of the paper is as follows: section 2 will introduce some preliminaries and in section 3, we have discussed about new hypothesis of fuzzy number. In section 4 , addition of two fuzzy numbers and its properties are discussed. In section 5, multiplications of two fuzzy numbers and its properties is demonstrated and finally the algebraic field structure of fuzzy real numbers is given in section 6 . In section 7, the new fuzzy number developed is used to solve fuzzy equation is demonstrated with an example. Section 8 is a conclusion and the paper ends by stating references.

\section{Preliminaries}

In this section, we will introduce some definitions.

Fuzzy set [6]: Let $U$ be any arbitrary set and $x$ be an element in $U$. Then a Fuzzy set $A$ is defined as $A=$ $\left\{\left(x, \mu_{A}(x)\right) / x \in E\right\}$, where $\mu_{A}(x)$ is the membership value of $x$ in $A$, which belongs to the closed interval $[0,1]$.

Normal fuzzy set [7]: A fuzzy set $A$ is said to be normal if its membership function $\mu_{A}(x)$ is unity, i.e. $\mu_{A}(x)=1$.

$\boldsymbol{\alpha}$ - cut [7]: An $\alpha-$ cut $\alpha_{A}$ of a fuzzy set $A$ is the set of all elements from the Universal set with membership value greater than $\alpha$ for $0 \leq \alpha \leq 1$. Thus it is defined as $\alpha_{A}=\left\{x \in E / \mu_{A}(x) \geq \alpha\right\}$.

Support of fuzzy set [7]: The support of a fuzzy set $A$ is denoted by $\operatorname{supp}(A)$ and is defined as the set of elements with membership having non zero value, i.e. $\sup p(A)=$ $\left\{x \in E / \mu_{A}(x)>0\right\}$.

Convex [7]: A fuzzy set $A$ is convex if $\mu_{A}\left(t x_{1}+(1-\right.$ $\left.t) x_{2}\right) \geq \min \left(\mu_{A}\left(x_{1}\right), \mu_{A}\left(x_{2}\right)\right), x_{1}, x_{2} \in E$ and $t \in[0,1]$. Moreover a fuzzy set is convex if all $\alpha$-level sets are convex.

Fuzzy Number [7]: A fuzzy set $A$ on $R$ must possess the following three properties to qualify as a fuzzy number

(1) $A$ must be a fuzzy normal set,

(2) $\alpha_{A}$ must be closed interval for every $\alpha \in[0,1]$,

(3) The support of a fuzzy set $A$ must be bounded.

Triangular fuzzy number [6]: A triangular fuzzy number is defined as $A=(a, b, c)$ for $a, b, c \in R$ and its membership is given by

$$
\mu_{A}(t)= \begin{cases}\frac{(t-a)}{(b-a)} & ; a \leq t \leq b \\ \frac{(c-t)}{(c-b)} & ; b \leq t \leq c \\ 0 & ; \text { otherwise }\end{cases}
$$

Diamond fuzzy number [6]: A diamond fuzzy number of a fuzzy set $A$ is defined as $A_{D}=\left\{a, b, c,\left(\alpha_{b}, \beta_{b}\right)\right\}$ and its membership is given by

$$
\mu_{A_{D}}(t)=\left\{\begin{array}{c}
0 \quad \text { for } t<a \\
\frac{t-a}{b-a} \quad \text { for } a \leq t \leq b \\
\frac{(c-t)}{(c-b)} \text { for } b \leq t \leq c \\
\alpha_{b}-\text { base } \\
\frac{a-t}{a-b} \quad \text { for } a \leq t \leq b \\
\frac{t-c}{b-c} \text { for } b \leq t \leq c \\
1 \quad \text { for } t=\beta_{b} \\
0 \text { otherwise }
\end{array}\right.
$$

where $\alpha_{b}$ is the base of the triangle $a \beta_{b} c$ and also for the inverted reflection of the above triangle.

\section{Fuzzy Number - A New Hypothesis}

In this section our objective is to give new hypothesis of fuzzy numbers and their representation. We introduce the concept of fuzzy number $n$, for any $n \in R$. The membership function $\mu$ of the fuzzy number $n$ has a domain which is a closed interval $[n-\epsilon, n+\delta]$ around $n$. However here in this work we define the membership functions as triangular; but it may be extended to a trapezoidal shape. We denote the fuzzy number $n$ by fuzzy $[n, \varepsilon, \delta, \lambda, \gamma]$ or simply by $\check{n}$. The arithmetic operations $(+,-, \times, /)$ are defined on two fuzzy numbers $\left[m, \epsilon_{1}, \delta_{1}, \lambda_{1}, \gamma_{1}\right]$ and $\left[n, \epsilon_{2}, \delta_{2}, \lambda_{2}, \gamma_{2}\right]$ by taking the special interval operations respectively on their fuzzy domains $\left[m-\epsilon_{1}, m+\delta_{1}\right]$ and $\left[n-\epsilon_{2}, n+\delta_{2}\right]$. We also show that they properly define the field structure in an extended sense.

\subsection{Hypothesis}

By a fuzzy number $n$ one should mean a number which is not expressed clearly as $n$; but which can be approximated with $n$, for the purpose specified in the application. This means a fuzzy $n$ is not "far away" from $n$, i.e. it is near to $n$ and can be considered as $n$ as far as the purpose of the application is concerned. Fuzzyness in the number $n$ means that it is near to $n$ and not very far away from $n$, because otherwise it is treated as quite different from $n$. How near or how far is dependent on the application. Generally we shall define this 'nearness' as less than or equal to 0.5. However this may be taken as big as per the demand of the user.

We shall define a fuzzy number $n(n \in R)$ as the collection of numbers close to $\mathrm{n}$ or $\mathrm{n}$ itself, and shall denote as $\bar{n}$.

\subsection{Definition: Fuzzy Number and its Membership Function}

For a number $n \in R$, we define fuzzy domain of $n$, denoted as $F D(n)$, as the collection of numbers very close to $n$ or itself and is given by the set $[n-\epsilon, n+\delta]$, where $\varepsilon$ and $\delta$ are non negative numbers less than or equal to some pre assigned non negative number $\alpha$. We will be taking $\alpha=0.5$ throughout the paper. 


\subsection{Membership Function}

Define $\mu: R \rightarrow[0,1]$, such that

$$
\mu(t)=\left\{\begin{array}{cl}
0 & ; \text { otherwise } \\
1+p^{\prime}(t-n) & ; n \leq t \leq n+\delta \\
1+p(t-n) & ; n-\epsilon \leq t \leq n
\end{array}\right.
$$

where $p=\frac{\lambda}{\epsilon}$ and $p^{\prime}=-\frac{\gamma}{\delta}$.

Here $\mu(n-\epsilon)=1-p \epsilon=1-\lambda$ and $\mu(n+\delta)=$ $1+p^{\prime} \delta=1-\gamma$.

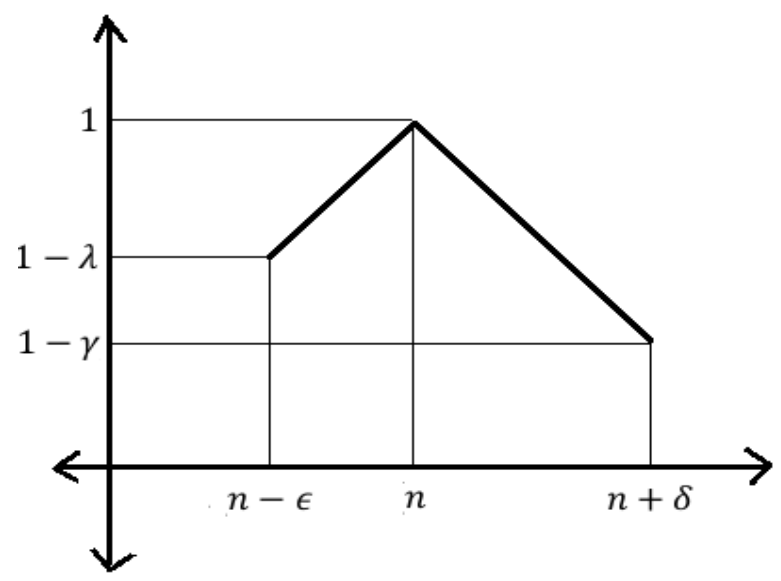

Figure 1. Graphical representation of fuzzy number $n$

Now, we recall $([n-\epsilon, n+\delta], \mu: R \rightarrow[0,1])$ a fuzzy number- $n$ and is denoted by $\widetilde{n}$.

Sometimes we write fuzzy number- $n$ as fuzzy $[n, \epsilon, \delta, \lambda, \gamma]$.

It is to be noted that the set $F D(n)$ (where $n \in R$ ) includes all real numbers, because $\varepsilon$ and $\delta$ are allowed to take value 0 .

When $\varepsilon=\delta=0$ for a fuzzy number $\widetilde{n}$, we shall write $F D(n)=[n-0, n+0]=n$.

Thus, we see that two fuzzy domains $\left[n-\epsilon_{1}, n+\delta_{1}\right]$ and $\left[n-\epsilon_{2}, n+\delta_{2}\right]$ can be treated as identical as there exists a proper homeomorphism between them for non zero values of $\epsilon_{1}, \delta_{1}, \epsilon_{2}$ and $\delta_{2}$.

Also note that when $n_{1}$ and $n_{2}$ are distinct, the two fuzzy domains $\left[n_{1}-\epsilon_{1}, n_{1}+\delta_{1}\right]$ and $\left[n_{2}-\epsilon_{2}, n_{2}+\delta_{2}\right]$ may or may not be disjoint.

\subsubsection{Theorem}

Given non zero $\epsilon_{1}, \delta_{1}, \epsilon_{2}$ and $\delta_{2}$ the function

$$
h_{n}:\left[n-\epsilon_{1}, n+\delta_{1}\right] \rightarrow\left[n-\epsilon_{2}, n+\delta_{2}\right]
$$

defined by

$$
h_{n}(x)=\left\{\begin{array}{lc}
\frac{\epsilon_{2}}{\epsilon_{1}}(x-n)+n & ; n-\epsilon_{1} \leq x \leq n \\
n & ; x=n \\
\frac{\delta_{2}}{\delta_{1}}(x-n)+n & ; n \leq x \leq n+\delta_{1}
\end{array}\right.
$$

is a homeomorphism between $\left[n-\epsilon_{1}, n+\delta_{1}\right]$ to $[n-$ $\left.\epsilon_{2}, n+\delta_{2}\right]$.

\section{Note:}

1. Now onwards $h_{r}$ shall denote this type of homeomorphism $h_{r}:\left[r-\epsilon_{1}, r+\delta_{1}\right] \rightarrow\left[r-\epsilon_{2}, r+\delta_{2}\right]$.

2 . Also see that two fuzzy $r$ can be treated as identical via Homeomorphism $h_{r}$.

\subsubsection{Theorem}

The fuzzy numbers $[m, \epsilon, \delta, \lambda, \gamma]$ and $\left[m, \epsilon^{\prime}, \delta^{\prime}, \lambda^{\prime}, \gamma^{\prime}\right]$ can be treated as identical.

We first see that by theorem 3.3.1, $[m-\epsilon, m+\delta]$ is the same as $\left[m-\epsilon^{\prime}, m+\delta^{\prime}\right]$.

Now, take the functions $\mu: R \rightarrow[0,1]$ and $\mu^{\prime}: R \rightarrow[0,1]$ given by

$$
\begin{gathered}
\mu(t)=\left\{\begin{array}{c}
1+\frac{\lambda}{\epsilon}(t-m) \text { if } m-\epsilon \leq t \leq \mathrm{m} \\
1-\frac{\gamma}{\delta}(t-m) \text { if } m \leq t \leq \mathrm{m}+\delta \text { and } \\
0 ; \quad \text { otherwise }
\end{array}\right. \\
\mu^{\prime}(t)=\left\{\begin{array}{c}
1+\frac{\lambda^{\prime}}{\epsilon^{\prime}}(t-m) \text { if } m-\epsilon^{\prime} \leq t \leq \mathrm{m} \\
1-\frac{\gamma^{\prime}}{\delta^{\prime}}(t-m) \text { if } m \leq t \leq \mathrm{m}+\delta^{\prime} \\
0 \quad ; \quad \text { otherwise }
\end{array}\right.
\end{gathered}
$$

We have the membership functions graphs of $\mu$ and $\mu^{\prime}$ as follows: 


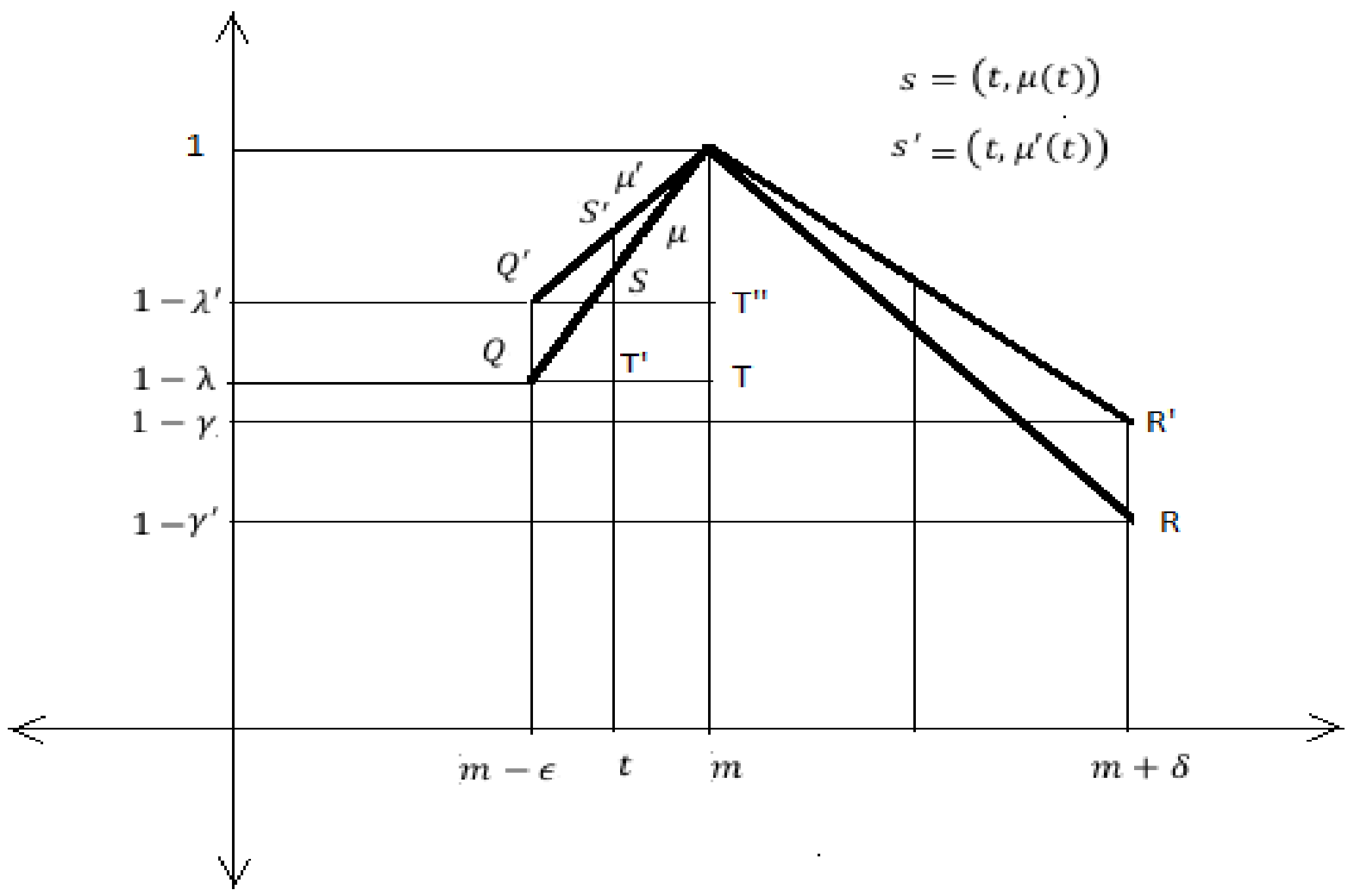

Figure 2. Graphical representation of fuzzy numbers

In $\triangle P Q Q^{\prime}$ and $\triangle P S S^{\prime}, Q Q^{\prime} \| S S^{\prime}$.

Therefore, $\frac{P S^{\prime}}{P Q^{\prime}}=\frac{P S}{P Q}=\frac{S S^{\prime}}{Q Q^{\prime}}$

$$
\begin{gathered}
\text { i.e. } \frac{P S^{\prime}}{P Q^{\prime}-P S^{\prime}}=\frac{P S}{P Q-P S}=\frac{S S^{\prime}}{Q Q^{\prime}-S S^{\prime}} \\
\text { i.e. } \frac{P Q^{\prime}}{Q^{\prime} S^{\prime}}=\frac{P S}{Q S}=\frac{S S^{\prime}}{\lambda^{\prime}-\lambda-S S^{\prime}}
\end{gathered}
$$

Similarly in $\triangle P Q T, S T^{\prime} \| P T$ and so

$$
\frac{P S}{Q S}=\frac{T T^{\prime}}{T^{\prime} Q}=\frac{m-t}{t-m+\epsilon}
$$

Similarly using the triangles $\triangle P Q^{\prime \prime} T^{\prime \prime}, S^{\prime} S \| P T^{\prime \prime}$, we obtain

$$
\frac{P S^{\prime}}{Q^{\prime} S^{\prime}}=\frac{m-t}{t-m+\epsilon}
$$

Now, using the equations (1), (2) and (3), we get as follows

$$
\frac{S^{\prime} S}{\lambda^{\prime}-\lambda-S S^{\prime}}=\frac{m-t}{t-m+\epsilon} \Rightarrow \frac{S^{\prime} S}{\lambda^{\prime}-\lambda}=\frac{m-t}{\epsilon} \Rightarrow S^{\prime} S=\frac{\lambda^{\prime}-\lambda}{\epsilon}(m-t) .
$$

This gives us the relation for each point $(t, \mu(t)) \rightarrow$ The unique point $\left(t, \mu^{\prime}(t)\right)$ which is given as

$$
\begin{gathered}
f[(t, \mu(t))]=\left[t, \mu(t)+\frac{\lambda^{\prime}-\lambda}{\epsilon}(m-t)\right] \\
\text { for } m-\epsilon \leq t \leq m
\end{gathered}
$$

$$
\mu(t)=\left\{\begin{array}{cc}
1+s(t-m) ; & \text { if } m-\epsilon \leq t \leq m \\
1+s^{\prime}(t-m) ; & \text { if } m \leq t \leq m+\delta \\
0 \quad ; & \text { Otherwise }
\end{array}\right.
$$$$
\text { where } s=\frac{\lambda}{\epsilon} \text { and } s^{\prime}=-\frac{\gamma}{\delta}
$$$$
\mu(t)=\left\{\begin{array}{cc}
1+s(t-m) ; & \text { if } m-\epsilon \leq t \leq m \\
1+s^{\prime}(t-m) ; & \text { if } m \leq t \leq m+\delta \\
0 \quad ; & \text { Otherwise }
\end{array}\right.
$$

$$
\text { where } s=\frac{\lambda}{\epsilon} \text { and } s^{\prime}=-\frac{\gamma}{\delta}
$$

$$
\mu^{\prime}(t)=\left\{\begin{array}{c}
1+r(t-m) ; \text { if } m-\epsilon^{\prime} \leq t \leq m \\
1+r^{\prime}(t-m) ; \quad \text { if } m \leq t \leq m+\delta^{\prime} \\
0 \quad ; \quad \text { Otherwise }
\end{array}\right.
$$

$$
\text { where } r=\frac{\lambda^{\prime}}{\epsilon^{\prime}} \text { and } r^{\prime}=-\frac{\gamma^{\prime}}{\delta^{\prime}}
$$

Now, from above

$$
\begin{gathered}
\mu(t)+\frac{\lambda^{\prime}-\lambda}{\epsilon}(m-t) \\
=\left\{\begin{array}{cc}
1+\frac{\lambda}{\epsilon}(t-m)+\frac{\lambda-\lambda^{\prime}}{\epsilon}(t-m) ; \text { if } & m-\epsilon \leq t \leq m \\
1-\frac{\gamma}{\delta}(t-m)+\frac{\gamma-\gamma^{\prime}}{\delta}(m-t) ; & \text { if } m \leq t \leq m+\delta \\
0 & ; \quad \text { otherwise }
\end{array}\right.
\end{gathered}
$$


which implies

$$
\begin{gathered}
1+\frac{\lambda}{\epsilon}(t-m+m-t)+\frac{\lambda^{\prime}}{\epsilon}(t-m) \\
=1+\frac{\lambda^{\prime}}{\epsilon}(t-m) .
\end{gathered}
$$

Thus, $\mu(t) \rightarrow \mu^{\prime}(t)$.

Similarly, we can write for $m \leq t^{\prime} \leq m+\delta$ such that

$$
(t, \mu(t)) \rightarrow\left(t, \mu(t)+\frac{\gamma-\gamma^{\prime}}{\delta}(t-m)\right) .
$$

By extending the domain of $f$ from $[m-\epsilon, m]$ to $[m-$ $\epsilon, m+\delta]=[m-\epsilon, m] \cup[m, m+\delta]$ and using the lemma, we map $(t, \mu(t))$ to $\left(t, \mu^{\prime}(t)\right)$ homeomorphically, for $m-\epsilon \leq t \leq m+\delta$. We have treated $[m-\epsilon, m+$ $\delta]$ and $\left[m-\epsilon^{\prime}, m+\delta^{\prime}\right]$ identical.

Thus the two fuzzy $m,[m, \epsilon, \delta, \lambda, \gamma]$ and $\left[m, \epsilon^{\prime}, \delta^{\prime}, \lambda^{\prime}, \gamma^{\prime}\right]$ can be treated as the same, as there is a special homeomorphism between the two.

\subsection{Equality of Two Fuzzy-n}

Two fuzzy numbers $\widetilde{n_{1}}$ and $\widetilde{n_{2}}$ are equal if and only if $n_{1}=n_{2}$.

\section{Addition of Two Fuzzy Numbers and Its Properties}

We define in this section the addition ' $+{ }^{\prime}$ of the two (nearly) defined fuzzy numbers. And we show that they maintain the usual algebraic structure of addition group $(R,+)$.

We define additions of two fuzzy numbers as follows:

Let $\quad F D(n)=\left[n-\epsilon_{1}, n+\delta_{1}\right] \quad$ and $\quad F D(m)=$ $\left[m-\epsilon_{2}, m+\delta_{2}\right]$ be any two fuzzy domains, then we define

$$
F D(m+n)=[(m+n)-\epsilon,(m+n)+\delta] .
$$

We can also define $F D(m)+F D(n)$ as

$$
\begin{aligned}
F D(m)+F D(n) & =\left[m-\epsilon_{2}, m+\delta_{2}\right]+\left[n-\epsilon_{1}, n+\delta_{1}\right] \\
& =[(m+n)-\epsilon,(m+n)+\delta]
\end{aligned}
$$

where

$$
\epsilon=\min \left\{\epsilon_{1}+\epsilon_{2}, 0.5\right\} \text { and } \delta=\min \left\{\delta_{1}+\delta_{2}, 0.5\right\} .
$$

From equations (4) and (5) and using theorem 3.3.1, $F D(m+n)=F D(m)+F D(n)$.
Now the membership function is defined as $\mu_{1}: F D(n) \rightarrow\left[1-\lambda_{1}, 1-\gamma_{1}\right]$ of $\breve{n}=\left[n, \epsilon_{1}, \delta_{1}, \lambda_{1}, \gamma_{1}\right]$. The graph of it is given in figure 3 .

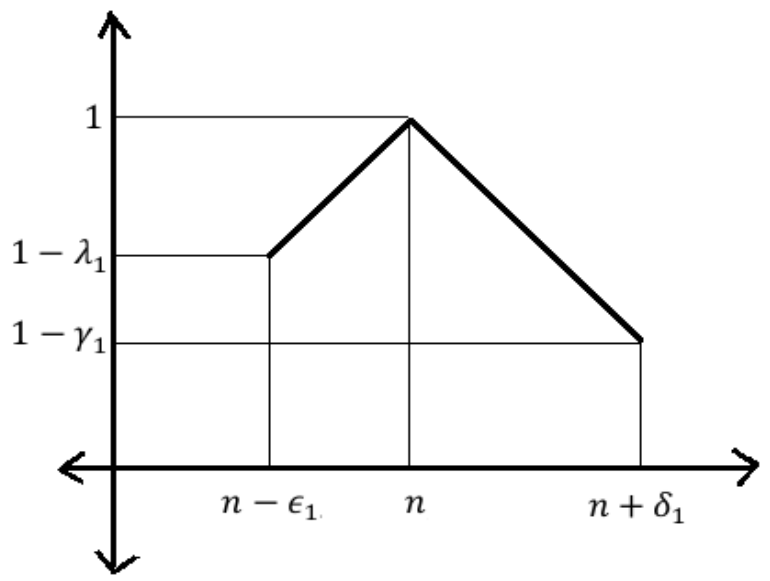

Figure 3. Graphical representation of fuzzy number $n$

Similarly, let $\mu_{2}: F D(m) \rightarrow\left[1-\lambda_{2}, 1-\gamma_{2}\right]$ of $\breve{m}=$ $\left[m, \epsilon_{2}, \delta_{2}, \lambda_{2}, \gamma_{2}\right]$ be defined whose graph is given as:

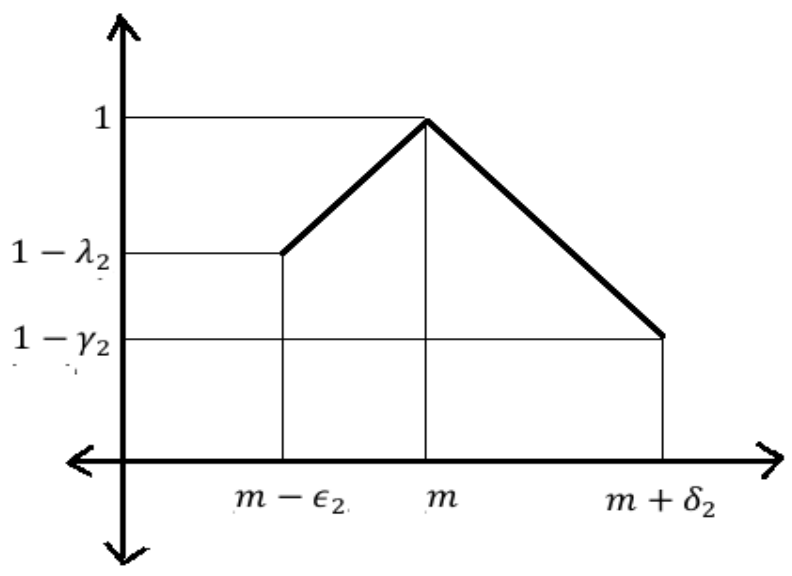

Figure 4. Graphical representation of fuzzy number $m$

We define the membership function of $F D(m+n)$ as given by $\mu: R \rightarrow[0,1]$, such that

$$
\left\{\begin{array}{cl}
1+p(t-(m+n)) ; & (m+n)-\epsilon \leq t \leq(m+n) \\
1+p^{\prime}(t-(m+n)) ; & (m+n) \leq t \leq(m+n)+\delta \\
0 \quad ; \text { otherwise }
\end{array}\right.
$$

where $p=\frac{\lambda}{\epsilon}$ and $p^{\prime}=-\frac{\gamma}{\delta}$. Then, $\mu((m+n)-\epsilon)=1-$ $p \epsilon=1-\lambda$ and $\mu((m+n)+\delta)=1+p^{\prime} \delta=1-\gamma$. 


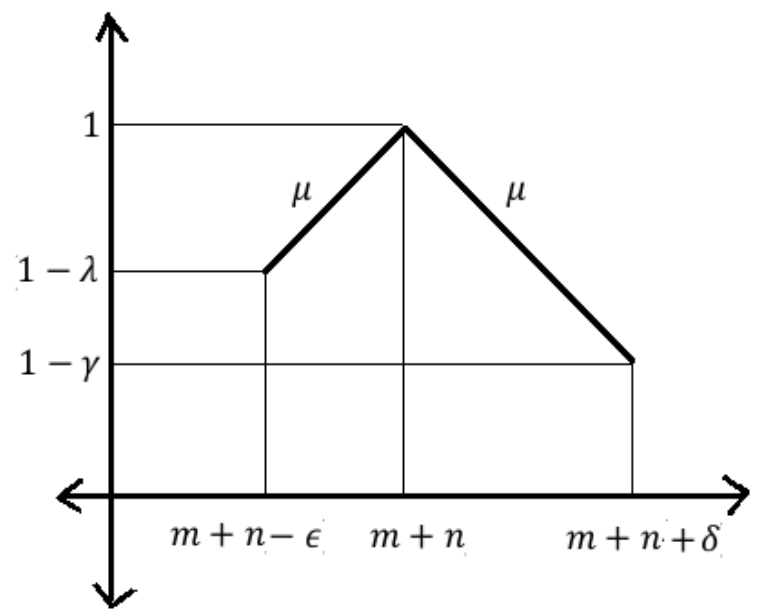

Figure 5. Graphical representation of fuzzy number $m+n$

Thus we have defined $\check{m}+\check{n}$ as $\overline{m+n}=[m+$ $n, \varepsilon, \delta, \lambda, \gamma]$, where

$\mu: R \rightarrow[0,1]$ as defined in (6) above on the fuzzy domain of the $F D(m+n)$.

\subsection{Properties for Addition of Fuzzy Numbers}

\subsubsection{Commutative Property}

Let $\check{n}=\left[n, \epsilon_{1}, \delta_{1}, \lambda_{1}, \gamma_{1}\right]$ and $\check{m}=\left[m, \epsilon_{2}, \delta_{2}, \lambda_{2}, \gamma_{2}\right]$.

For $n, m \in R$, let $F D(n)=\left[n-\epsilon_{1}, n+\delta_{1}\right]$ and $F D(m)=\left[m-\epsilon_{2}, m+\delta_{2}\right]$. Then

$F D(n+m)=[(n+m)-\epsilon,(n+m)+\delta]$ where $\epsilon=\min \left\{\epsilon_{1}+\epsilon_{2}, 0.5\right\}$ and

$$
\begin{gathered}
\delta=\min \left\{\delta_{1}+\delta_{2}, 0.5\right\} . \\
=[(n+m)-\epsilon,(n+m)+\delta]
\end{gathered}
$$

Now,

$$
\begin{gathered}
F D(m+n)=[(m+n)-\epsilon,(m+n)+\delta] \text { where } \\
\epsilon=\min \left\{\epsilon_{2}+\epsilon_{1}, 0.5\right\} \& \\
\delta=\min \left\{\delta_{2}+\delta_{1}, 0.5\right\} . \\
=[(n+m)-\epsilon,(n+m)+\delta]
\end{gathered}
$$

Therefore, we get

$$
F D(m+n)=F D(n+m) \text {. }
$$

We observe that, obviously, the membership function on both the side i.e.FD $(m+n)$ and $F D(n+m)$ are the same or can be considered the same using the techniques of the earlier theorem 3.3.2.

Hence, $\check{n}+\check{m}=\check{m}+\check{n}$.

\subsubsection{Associative Property}

For $n, m, r \in R$, let $(n)=\left[n-\epsilon_{1}, n+\delta_{1}\right], F D(m)=$ $\left[m-\epsilon_{2}, m+\delta_{2}\right]$ and $F D(r)=\left[r-\epsilon_{3}, r+\delta_{3}\right]$, then

$$
\left[(n+m)-\epsilon_{,}(n+m)+\delta\right]+\left[r-\epsilon_{3}, r+\delta_{3}\right],
$$

where $\epsilon=\min \left\{\epsilon_{1}+\epsilon_{2}, 0.5\right\}$ and $\delta=\min \left\{\delta_{1}+\delta_{2}, 0.5\right\}$.

$$
\begin{gathered}
F D(n+m)+F D(r)=\left[(n+m)+r-\varepsilon^{\prime},(n+m)+\right. \\
\left.r+\delta^{\prime}\right] .
\end{gathered}
$$

$$
\begin{aligned}
& \text { where } \epsilon^{\prime}=\min \left\{\epsilon+\epsilon_{3}, 0.5\right\}=\min \left\{\epsilon_{1}+\epsilon_{2}+\epsilon_{3}, 0.5\right\} \\
& =\min \left\{\begin{array}{l}
\epsilon_{1}+\epsilon_{2}+\epsilon_{3} \text { if } \epsilon_{1}+\epsilon_{2}+\epsilon_{3}<0.5 \\
0.5 \quad \text { if } \epsilon_{1}+\epsilon_{2}+\epsilon_{3} \geq 0.5
\end{array}\right. \text { and } \\
& \delta^{\prime}=\min \left\{\delta+\delta_{3}, 0.5\right\}=\min \left\{\delta_{1}+\delta_{2}+\delta_{3}, 0.5\right\} \\
& =\min \left\{\begin{array}{l}
\delta_{1}+\delta_{2}+\delta_{3} \text { if } \delta_{1}+\delta_{2}+\delta_{3}<0.5 \\
0.5 \quad \text { if } \delta_{1}+\delta_{2}+\delta_{3} \geq 0.5
\end{array}\right. \\
& F D(n+(m+r)) \\
& =\left[n+(m+r)-\varepsilon^{\prime}, n+(m+r)+\delta^{\prime}\right] \\
& F D(n)+F D(m+r)=\left[n-\epsilon_{1}, n+\delta_{1}\right]+[(m+r)- \\
& \epsilon,(m+r)+\delta]
\end{aligned}
$$

where $\epsilon=\min \left\{\epsilon_{2}+\epsilon_{3}, 0.5\right\}$ and $\delta=\min \left\{\delta_{2}+\delta_{3}, 0.5\right\}$.

$$
=\left[n+(m+r)-\varepsilon^{\prime}, n+(m+r)+\delta^{\prime}\right]
$$

and $\epsilon^{\prime}=\min \left\{\epsilon_{1}+\epsilon, 0.5\right\}=\min \left\{\epsilon_{1}+\epsilon_{2}+\epsilon_{3}, 0.5\right\}$

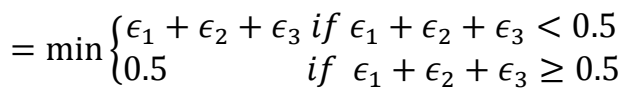

$$
\begin{aligned}
& \delta^{\prime}=\min \left\{\delta_{1}+\delta, 0.5\right\} \\
& =\min \left\{\begin{array}{l}
\delta_{1}+\delta_{2}+\delta_{3} \text { if } \delta_{1}+\delta_{2}+\delta_{3}<0.5 \\
0.5 \quad \text { if } \delta_{1}+\delta_{2}+\delta_{3} \geq 0.5
\end{array}\right. \\
& =F D(n+(m+r))
\end{aligned}
$$

From equations (9) and (10) and by using theorem 3.3.2, we have

$$
(\tilde{n}+\tilde{m})+\tilde{r}=\tilde{n}+(\tilde{m}+\tilde{r})
$$

We observe that the two membership functions on both sides can be shown to the same or identical using the same techniques as that demonstrated in the theorem 3.3.2.

Note: We would like to avoid the repetitions of the same type of remarks in the following. Therefore we simply put remark at necessary stage that using theorem 3.3.2 it can be shown that the two concerned fuzzy numbers are the same.

\subsubsection{Additive Identity}

For $0 \in R$, we define $F D(0)=\left[0-\epsilon_{2}, 0+\delta_{2}\right]$.

Now $F D(n)+F D(0)=\left[n-\epsilon_{1}, n+\delta_{1}\right]+\left[0-\epsilon_{2}, 0+\right.$ $\left.\delta_{2}\right]$

$$
=[n+0-\epsilon, n+0+\delta]
$$

where $\epsilon=\min \left\{\epsilon_{1}+\epsilon_{2}, 0.5\right\}$ and $\delta=\min \left\{\delta_{1}+\delta_{2}, 0.5\right\}$

$$
\begin{gathered}
=[n-\epsilon, n+\delta] \\
=F D(n) .
\end{gathered}
$$

Now,

$$
\begin{gathered}
F D(0)+F D(n)=\left[0-\epsilon_{2}, 0+\delta_{2}\right]+\left[n-\epsilon_{1}, n+\delta_{1}\right] \\
=[0+n-\epsilon, 0+n+\delta]
\end{gathered}
$$

where $\epsilon=\min \left\{\epsilon_{2}+\epsilon_{1}, 0.5\right\}$ and $\delta=\min \left\{\delta_{2}+\delta_{1}, 0.5\right\}$

$$
\begin{gathered}
=[n-\epsilon, n+\delta] \\
=F D(n) .
\end{gathered}
$$

It can be shown that $\tilde{n}+\tilde{0}=\tilde{0}+\tilde{n}=\tilde{n}$

Thus $\widetilde{0}$ is the additive identity for fuzzy number. 


\subsubsection{Additive Inverse}

For each $F D(n)=\left[n-\epsilon_{1}, n+\delta_{1}\right]$, we define $F D(-n)=$ $\left[-n-\epsilon_{2},-n+\delta_{2}\right]$, then

$$
\begin{aligned}
& F D(n)+F D(-n) \\
= & {\left[n-\epsilon_{1}, n+\delta_{1}\right]+\left[-n-\epsilon_{2},-n+\delta_{2}\right] }
\end{aligned}
$$

where $\epsilon=\min \left\{\epsilon_{1}+\epsilon_{2}, 0.5\right\}$ and $\delta=\min \left\{\delta_{1}+\delta_{2}, 0.5\right\}$

$$
\begin{gathered}
=[n+(-n)-\epsilon, n+(-n)+\delta] \\
=[0-\epsilon, 0+\delta] \\
=F D(0) .
\end{gathered}
$$

i.e. $F D(-n)$ is the additive inverse of $F D(n)$.

It is noted that

$$
F D(-n)=\left[-n-\epsilon_{2},-n+\delta_{2}\right]
$$

and

$$
-F D(n)=-\left[n-\epsilon_{1}, n+\delta_{1}\right]=\left[-n-\delta_{1},-n+\epsilon_{1}\right]
$$

From (11) and (12) and by Theorem 3.3.2, we have

$$
F D(-n)=-F D(n) .
$$

Thus, we define

$$
-\check{n}=-\left[n, \epsilon_{1}, \delta_{1}, \lambda_{1}, \gamma_{1}\right]=\left[-n, \epsilon_{2}, \delta_{2}, \lambda_{2}, \gamma_{2}\right]=-\bar{n} .
$$

\subsection{Subtraction of Two Fuzzy Numbers}

We define subtraction of two fuzzy numbers as follows:

Let $\quad F D(n)=\left[n-\epsilon_{1}, n+\delta_{1}\right] \quad$ and $\quad F D(m)=$ $\left[m-\epsilon_{2}, m+\delta_{2}\right]$ are any two fuzzy domains then we define

$$
\begin{gathered}
{\left[m-\epsilon_{2}, m+\delta_{2}\right]-\left[n-\epsilon_{1}, n+\delta_{1}\right]} \\
\quad=[(m-n)-\epsilon,(m-n)+\delta]
\end{gathered}
$$

where $\epsilon=\min \left\{\epsilon_{1}+\epsilon_{2}, 0.5\right\}$ and $\delta=\min \left\{\delta_{1}+\delta_{2}, 0.5\right\}$.

We write $F D(m-n)=[(m-n)-\epsilon,(m-n)+\delta]$

Now let $\lambda \leq \min \left\{\epsilon_{1}, \epsilon_{2}\right\}$ and $\gamma \leq \min \left\{\delta_{1}, \delta_{2}\right\}$.

The membership function on $F D(m-n)$ is defined as $\mu: R \rightarrow[0,1]$, such that

$$
\begin{gathered}
\mu(t)= \\
\left\{\begin{array}{cc}
1+p(t-(m-n)) ; & (m-n)-\epsilon \leq t \leq(m-n) \\
1+p^{\prime}(t-(m-n)) ; & (m-n) \leq t \leq(m-n)+\delta \\
0 \quad ; \quad \text { otherwise }
\end{array}\right.
\end{gathered}
$$

where $p=\frac{\lambda}{\epsilon}$ and $p^{\prime}=-\frac{\gamma}{\delta}$, then we see that

$$
\begin{gathered}
\mu((m-n)-\epsilon)=1-p \epsilon=1-\lambda \& \mu((m-n)+\delta)= \\
1+p^{\prime} \delta=1-\gamma
\end{gathered}
$$

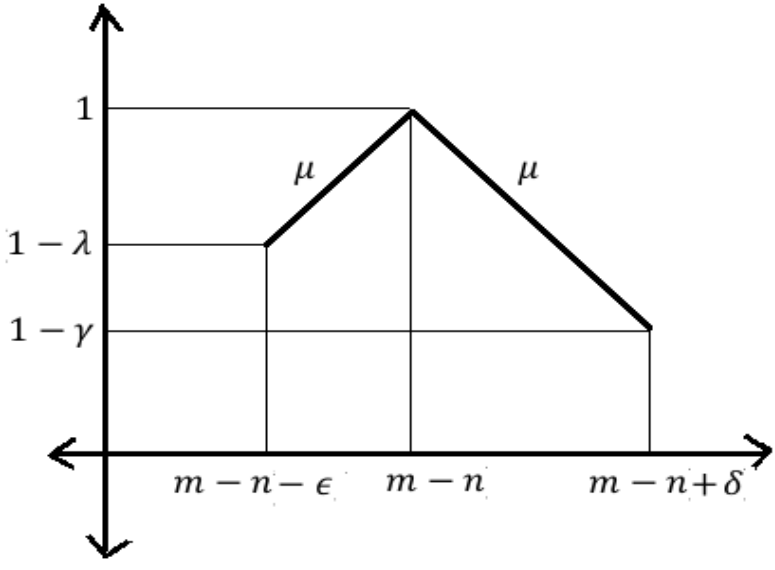

Figure 6. Graphical representation of fuzzy number $m-n$

Thus we define the $\breve{m}-\check{n}$ as the fuzzy number [ $m-$ $n, \varepsilon, \delta, \lambda, \gamma]$ denoted as $\overline{m-n}$, as defined by (14).

\section{Multiplication of Two Fuzzy Numbers}

Let $\quad F D(n)=\left[n-\epsilon_{1}, n+\delta_{1}\right] \quad$ and $\quad F D(m)=$ $\left[m-\epsilon_{2}, m+\delta_{2}\right]$ be any two domains of fuzzy $\breve{n}=$ $\left[n, \epsilon_{1}, \delta_{1}, \lambda_{1}, \gamma_{1}\right]$ and $\breve{m}=\left[m, \epsilon_{2}, \delta_{2}, \lambda_{2}, \gamma_{2}\right]$ respectively, then we define the multiplication of $\widetilde{n}$ and $\widetilde{m}$ as follows:

$$
\begin{aligned}
F D(n) \cdot F D(m) & =\left[n-\epsilon_{1}, n+\delta_{1}\right]\left[m-\epsilon_{2}, m+\delta_{2}\right] \\
& =[n m-\epsilon, n m+\delta]
\end{aligned}
$$

where $\epsilon=\min \left\{\epsilon_{1} \epsilon_{2}, 0.5\right\}$ and $\delta=\min \left\{\delta_{1} \delta_{2}, 0.5\right\}$.

Also note that

$$
F D(n m)=\left[n m-\epsilon^{\prime}, n m+\delta^{\prime}\right] .
$$

From equations (15), (16) and Theorem 3.3.2, we have

$$
F D(n) F D(m)=F D(n m)
$$

Now we define the membership function on $F D(n)$, $F D(m)$ on $\mu: R \rightarrow[0,1]$ and is given by

$\mu(t)=\left\{\begin{array}{cc}1+p(t-(\mathrm{nm})) ; & (\mathrm{nm})-\epsilon \leq t \leq(\mathrm{nm}) \\ 1+p^{\prime}(t-(\mathrm{nm})) ; & (\mathrm{nm}) \leq t \leq(\mathrm{nm})+\delta \\ 0 \quad ; \text { otherwise }\end{array}\right.$

where $\quad p=\frac{\lambda}{\epsilon}, p^{\prime}=-\frac{\gamma}{\delta}$ and $\lambda \geq \max \left\{\lambda_{1}, \lambda_{2}\right\}, \gamma \geq$ $\max \left\{\gamma_{1}, \gamma_{2}\right\}$, then

$$
\begin{gathered}
\mu((\mathrm{nm})-\epsilon)=1-p \epsilon=1-\lambda \text { and } \mu((\mathrm{nm})+\delta)= \\
1+p^{\prime} \delta=1-\gamma
\end{gathered}
$$




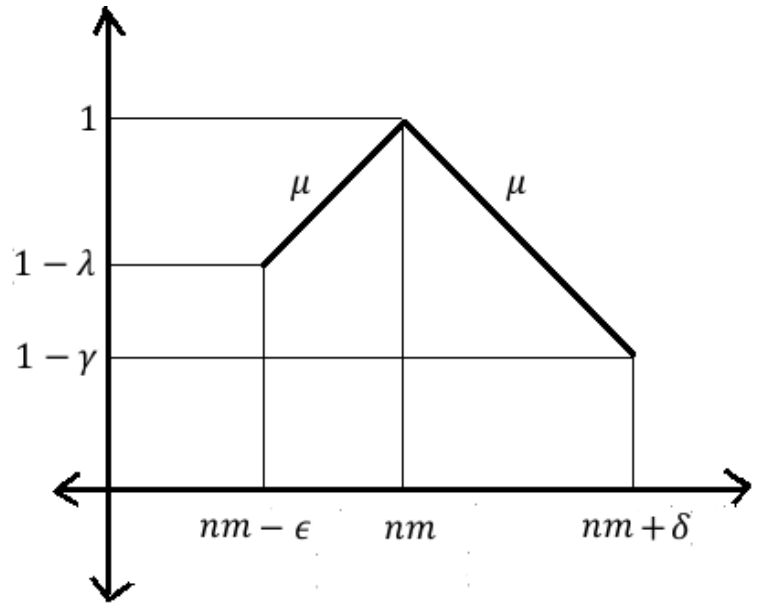

Figure 7. Graphical representation of fuzzy number $\mathrm{nm}$.

Thus the fuzzy number $\tilde{n} \tilde{m}=[\mathrm{nm}, \varepsilon, \delta, \lambda, \gamma]$ on $F D(n) F D(m)=[n m-\epsilon, n m+\delta]$ is defined as the fuzzy number

$\widetilde{n m}=\left[n m, \varepsilon^{\prime}, \delta^{\prime}, \lambda^{\prime}, \gamma^{\prime}\right]$ with the membership function

$$
\mu^{\prime}(t)=\left\{\begin{array}{cc}
1+q(t-(\mathrm{nm})) ; & (\mathrm{nm})-\epsilon^{\prime} \leq t \leq(\mathrm{nm}) \\
1+q^{\prime}(t-(\mathrm{nm})) ; & (\mathrm{nm}) \leq t \leq(\mathrm{nm})+\delta^{\prime} \\
0 \quad ; \text { otherwise }
\end{array}\right.
$$

where $q=\frac{\lambda \prime}{\epsilon \prime}$ and $q^{\prime}=-\frac{\gamma^{\prime}}{\delta^{\prime}}$.

$$
\text { i.e. } \tilde{n} \widetilde{m}=\tilde{n m}
$$

\subsection{Properties for Multiplication of Fuzzy Numbers}

\subsubsection{Commutative Property:}

Let $(n)=\left[n-\epsilon_{1}, n+\delta_{1}\right], F D(m)=\left[m-\epsilon_{2}, m+\right.$ $\left.\delta_{2}\right]$, and $n, m \in R$, then $F D(n) F D(m)=F D(n m)$ as discussed earlier.

Thus, $F D(n) F D(m)$ is defined as

$$
\begin{aligned}
& F D(n) F D(m)=\left[n-\epsilon_{1}, n+\delta_{1}\right]\left[m-\epsilon_{2}, m+\delta_{2}\right] \\
& =[n m-\epsilon, n m+\delta], \\
& \text { where } \epsilon=\min \left\{\epsilon_{1} \epsilon_{2}, 0.5\right\} \text { and } \delta=\min \left\{\delta_{1} \delta_{2}, 0.5\right\} \\
& =[m n-\epsilon, m n+\delta] \\
& \begin{aligned}
F D(m) F D(n) & =\left[m-\epsilon_{2}, m+\delta_{2}\right]\left[n-\epsilon_{1}, n+\delta_{1}\right] \\
& =[m n-\epsilon, m n+\delta],
\end{aligned}
\end{aligned}
$$

where

$$
\epsilon=\min \left\{\epsilon_{1} \epsilon_{2}, 0.5\right\} \text { and } \delta=\min \left\{\delta_{1} \delta_{2}, 0.5\right\} .
$$

From equations (18) and (19), we have $F D(n) F D(m)=$ $F D(m) F D(n)$,

i.e. $F D(n m)=F D(n) F D(m)=F D(m) F D(n)=F D(m n)$ and so obviously we have

$$
\widetilde{m n}=[m n, \varepsilon, \delta, \lambda, \gamma]=\left[n m, \varepsilon^{\prime}, \delta^{\prime}, \lambda^{\prime}, \gamma^{\prime}\right]=\widetilde{n m} .
$$

\subsubsection{Associative Property}

Let $n, m, r \in R \quad$ and $F D(n)=\left[n-\epsilon_{1}, n+\delta_{1}\right]$, $F D(m)=\left[m-\epsilon_{2}, m+\delta_{2}\right], F D(r)=\left[r-\epsilon_{3}, r+\delta_{3}\right]$ are three fuzzy domains of fuzzy numbers $\tilde{n}=\left[n, \epsilon_{1}, \delta_{1}, \lambda_{1}, \gamma_{1}\right]$, $\widetilde{m}=\left[m, \epsilon_{2}, \delta_{2}, \lambda_{2}, \gamma_{2}\right] \quad$ and $\quad \tilde{r}=\left[r, \epsilon_{3}, \delta_{3}, \lambda_{3}, \gamma_{3}\right]$ respectively, then

$F D(n m) F D(r)=\left[n m-\epsilon^{\prime}, n m+\delta^{\prime}\right]\left[r-\epsilon_{3}, r+\delta_{3}\right]$

where $\epsilon=\min \left\{\epsilon_{1} \epsilon_{2}, 0.5\right\}$ and $\delta=\min \left\{\delta_{1} \delta_{2}, 0.5\right\}$.

Thus, $(\mathrm{nm}) F D(r)=[(\mathrm{nm}) r-\epsilon,(\mathrm{nm}) r+\delta]$,

where $\epsilon=\min \left\{\epsilon^{\prime} \epsilon_{3}, 0.5\right\}$ and $\delta=\min \left\{\delta^{\prime} \delta_{3}, 0.5\right\}$

$$
=[n(m r)-\epsilon, n(m r)+\delta]
$$

Now,

$F D(n) F D(m r)=\left[n-\epsilon_{1}, n+\delta_{1}\right]\left[n m-\epsilon^{\prime}, n m+\delta^{\prime}\right]$ where

$$
\begin{gathered}
\epsilon^{\prime}=\min \left\{\epsilon_{2} \epsilon_{3}, 0.5\right\} \text { and } \delta^{\prime}=\min \left\{\delta_{2} \delta_{3}, 0.5\right\} \\
=\left[n(m r)-\epsilon^{\prime \prime \prime}, n(m r)+\delta^{\prime \prime \prime}\right]
\end{gathered}
$$

where $\epsilon^{\prime \prime \prime}=\min \left\{\epsilon_{1} \epsilon^{\prime}, 0.5\right\}$ and $\delta^{\prime \prime \prime}=\min \left\{\delta_{3} \delta^{\prime}, 0.5\right\}$.

Since for any real number $n, m, r$, we have $(n m) r=$ $n(m r)$ by using theorem 3.3.2 and equations (19), (20), we have

$$
(\tilde{n} \tilde{m}) \tilde{r}=\tilde{n}(\tilde{m} \tilde{r}) .
$$

\subsubsection{Multiplicative Identity}

For $1 \in R$, we have $\tilde{1}=\left[1, \epsilon_{2}, \delta_{2}, \lambda_{2}, \gamma_{2}\right]$. Using multiplicative identity on any fuzzy number $\tilde{n}=$ $\left[n, \epsilon_{1}, \delta_{1}, \lambda_{1}, \gamma_{1}\right]$, we have

$$
\begin{gathered}
F D(n) F D(1)=\left[n-\epsilon_{1}, n+\delta_{1}\right]\left[1-\epsilon_{2}, 1+\delta_{2}\right] \\
=[n 1-\epsilon, n 1+\delta] \text {, where } \epsilon=\min \left\{\epsilon_{1} \epsilon_{2}, 0.5\right\} \\
\text { and } \delta=\min \left\{\delta_{1} \delta_{2}, 0.5\right\} \\
=[n-\epsilon, n+\delta]
\end{gathered}
$$

Now, since $\left[n-\epsilon_{1}, n+\delta_{1}\right]{ }_{n}^{h_{n}}[n-\epsilon, n+\delta]$, i.e. both the closed intervals are identical via the homeomorphism $h_{n}$, hence we can write

$$
\begin{gathered}
{\left[n-\epsilon_{1}, n+\delta_{1}\right] \cong[n-\epsilon, n+\delta] .} \\
\text { i.e. } F D(n) F D(1)=F D(n) .
\end{gathered}
$$

Similarly, we have $F D(1) F D(n)=F D(n)$.

Thus $F D(1)$ is the multiplicative identity for fuzzy domains.

Hence, we can show that

$$
\begin{aligned}
& \tilde{n} \tilde{1}=\left[n, \epsilon_{1}, \delta_{1}, \lambda_{1}, \gamma_{1}\right]\left[1, \epsilon_{2}, \delta_{2}, \lambda_{2}, \gamma_{2}\right] \\
= & {\left[1, \epsilon_{2}, \delta_{2}, \lambda_{2}, \gamma_{2}\right]\left[n, \epsilon_{1}, \delta_{1}, \lambda_{1}, \gamma_{1}\right]=\tilde{1} \tilde{n} . }
\end{aligned}
$$

i.e. $\tilde{1}$ is the multiplicative identity of fuzzy numbers.

\subsubsection{Multiplicative Inverse}

For each non-zero $n \in R$, we define fuzzy $-\frac{1}{n}$ denoted by $\left(\frac{1}{n}\right)$ as

$$
\left(\frac{\widetilde{1}}{n}\right)=\left[\frac{1}{n}, \epsilon_{2}, \delta_{2}, \lambda_{2}, \gamma_{2}\right]
$$

Then 


$$
\begin{gathered}
\widetilde{n}\left(\widetilde{\frac{1}{n}}\right)=\left[n, \epsilon_{1}, \delta_{1}, \lambda_{1}, \gamma_{1}\right]\left[\frac{1}{n}, \epsilon_{2}, \delta_{2}, \lambda_{2}, \gamma_{2}\right] \\
=[1, \varepsilon, \delta, \lambda, \gamma] \\
=\widetilde{1}
\end{gathered}
$$

Thus $\left(\frac{\widetilde{1}}{n}\right)$ is the multiplicative inverse of $\tilde{n}$.

\subsection{Division of Two Fuzzy Numbers}

Let $\quad F D(n)=\left[n-\epsilon_{1}, n+\delta_{1}\right] \quad$ and $\quad F D(m)=$ $\left[m-\epsilon_{2}, m+\delta_{2}\right]$ be any two fuzzy domains such that $m \neq 0$, then we define division of fuzzy domains as follows:

$$
\frac{F D(n)}{F D(m)}=\frac{\left[n-\epsilon_{1}, n+\delta_{1}\right]}{\left[m-\epsilon_{2}, m+\delta_{2}\right]}=\left[\frac{n}{m}-\epsilon, \frac{n}{m}+\delta\right]
$$

where $\epsilon=\min \left\{\epsilon_{1} \epsilon_{2}, 0.5\right\}$ and $\delta=\min \left\{\delta_{1} \delta_{2,}, 0.5\right\}$.

Note that for $m \neq 0$ we have

$$
F D\left(\frac{n}{m}\right)=\left[\frac{n}{m}-\epsilon^{\prime}, \frac{n}{m}+\delta^{\prime}\right]
$$

where $\left|\epsilon^{\prime}\right| \leq 0.5$ and $\left|\delta^{\prime}\right| \leq 0.5$.

Now, since $\left[\frac{n}{m}-\epsilon, \frac{n}{m}+\delta\right] \stackrel{h_{n}}{\sim}\left[\frac{n}{m}-\epsilon^{\prime}, \frac{n}{m}+\delta^{\prime}\right]$, i.e. both the closed intervals are identical via the homeomorphism $h_{n}$, we can write

$$
\left[\frac{n}{m}-\epsilon, \frac{n}{m}+\delta\right] \cong\left[\frac{n}{m}-\epsilon^{\prime}, \frac{n}{m}+\delta^{\prime}\right] .
$$

In this sense, $\frac{F D(n)}{F D(m)}=F D\left(\frac{n}{m}\right)$.

Using theorem 3.3.2, we have

$\frac{\widetilde{n}}{\widetilde{m}}=\left(\frac{\widetilde{n}}{m}\right)=\left[\frac{n}{m}, \varepsilon, \delta, \lambda, \gamma\right]$, where the membership function of $\left(\frac{\pi}{m}\right)$ is given by $\mu: R \rightarrow[0,1]$ such that

$$
\mu(t)=\left\{\begin{array}{cl}
1+p\left(t-\left(\frac{n}{m}\right)\right) ; & \left(\frac{n}{m}\right)-\epsilon \leq t \leq\left(\frac{n}{m}\right) \\
1+p^{\prime}\left(t-\frac{n}{m}\right) ; & \left(\frac{n}{m}\right) \leq t \leq\left(\frac{n}{m}\right)+\delta \\
0 \quad ; \text { otherwise }
\end{array}\right.
$$

where $\quad p=\frac{\lambda}{\epsilon} \quad, \quad p^{\prime}=-\frac{\gamma}{\delta} \quad, \quad \lambda \geq \max \left\{\lambda_{1}, \lambda_{2}\right\}, \gamma \geq$ $\max \left\{\gamma_{1}, \gamma_{2}\right\}, \mu\left(\left(\frac{n}{m}\right)-\epsilon\right)=1-p \epsilon=1-\lambda$ and $\mu\left(\left(\frac{n}{m}\right)+\right.$ $\delta)=1+p^{\prime} \delta=1-\gamma$.

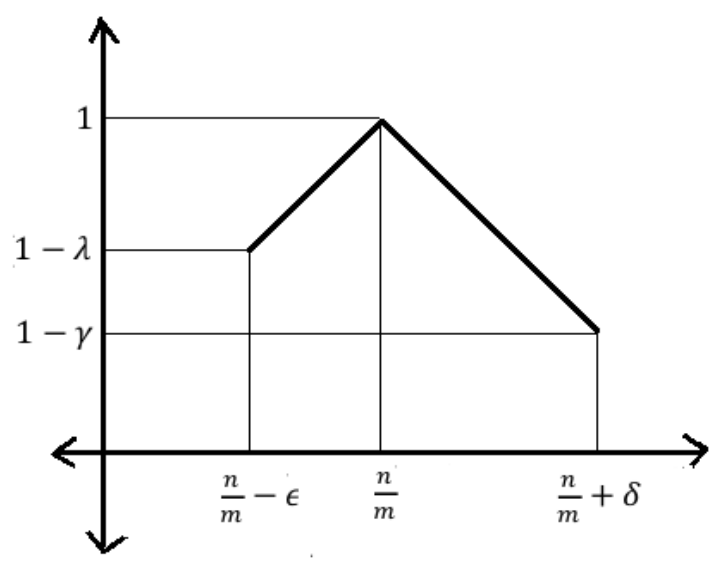

Figure 8. Graphical representation of fuzzy number $\frac{n}{m}$.
Now we recall $\left(\left[\frac{n}{m}-\epsilon, \frac{n}{m}+\delta\right], \mu: R \rightarrow[0,1]\right)$ as defined above in (23), division of two fuzzy numbers is denoted by $\left(\frac{\widetilde{n}}{m}\right)$.

Thus we can also define $\frac{\widetilde{n}}{\widetilde{m}}=\overline{\left(\frac{n}{m}\right)}$, where $m \neq 0$.

\subsection{Theorem}

Fuzzy number multiplication is distributive over fuzzy number addition.

For three fuzzy numbers $\widetilde{n}=\left[n, \epsilon_{1}, \delta_{1}, \lambda_{1}, \gamma_{1}\right]$, $\widetilde{m}=\left[m, \epsilon_{2}, \delta_{2}, \lambda_{2}, \gamma_{2}\right]$ and $\widetilde{r}=\left[r, \epsilon_{3}, \delta_{3}, \lambda_{3}, \gamma_{3}\right]$, we have $\widetilde{n}(\widetilde{m}+\widetilde{r})=\widetilde{n} \widetilde{m}+\widetilde{n} \widetilde{r}$. It has a trivial proof so we are omitting it here.

\section{The Algebraic Field Structure of Fuzzy Real Numbers}

\subsection{Introduction}

The set of fuzzy numbers $\widetilde{R}=\{\widetilde{r} / r \in R\}$ supports the structure of addition and multiplication of the usual reals in $R$ in a similar fashion in the sense (via homeomorphism $h_{r}$ ) as discussed above.

Now let $\bar{r}=[\tilde{r}]_{\varepsilon}$ denote the collection of all $\widetilde{r}=[r-$ $\left.\epsilon_{1}, r+\epsilon_{2}\right]$, such that $\left|\epsilon_{1}\right| \leq 0.5$ and $\left|\epsilon_{2}\right| \leq 0.5$,

i.e. $\bar{r}=[\widetilde{r}]_{\varepsilon}=\left\{\left[r-\epsilon_{1}, r+\epsilon_{2}\right] /\left|\epsilon_{1}\right| \leq 0.5\right.$ and $\left.\left|\epsilon_{2}\right| \leq 0.5, r \in R\right\}$.

Let $R_{\varepsilon}=\left\{\bar{r}=[\widetilde{r}]_{\varepsilon} / r \in R\right\}$. Now we define fuzzy algebraic operations,,$+- \times$ and / on the set

$R_{\varepsilon}=\left\{\bar{r}=[\widetilde{r}]_{\varepsilon} / r \in R\right\}$ as follows:

\subsection{Algebraic Operations on $R_{\varepsilon}$}

For $R_{\varepsilon}=\left\{\bar{r}=[\widetilde{r}]_{\varepsilon}, r \in R\right\}$, let $\overline{r_{1}}, \overline{r_{2}} \in R_{\varepsilon}$, we define the algebraic operations as follows:

1) $\overline{r_{1}}+\overline{r_{2}}=\left[\widetilde{r_{1}}\right]_{\varepsilon}+\left[\widetilde{r_{2}}\right]_{\varepsilon}=\left[\widetilde{r_{1}+r_{2}}\right]_{\varepsilon}=\overline{r_{1}+r_{2}}$ where $\widetilde{r_{1}}+\widetilde{r_{2}}=\left[r_{1}, \epsilon_{1}, \delta_{1}, \lambda_{1}, \gamma_{1}\right]+\left[\underline{r_{2}+\epsilon_{2}}, \delta_{2}, \lambda_{2}, \gamma_{2}\right]=$ $\left[r_{1}+r_{2}, \epsilon, \delta, \lambda, \gamma\right]=r_{1}+r_{2}$

2) $\overline{r_{1}}-\overline{r_{2}}=\left[\widetilde{r_{1}}\right]_{\varepsilon}-\left[\widetilde{r_{2}}\right]_{\varepsilon}=\left[\widetilde{r_{1}-r_{2}}\right]_{\varepsilon}=\overline{r_{1}-r_{2}}$ where $\widetilde{r_{1}}-\widetilde{r_{2}}=\left[r_{1}, \epsilon_{1}, \delta_{1}, \lambda_{1}, \gamma_{1}\right]-\left[r_{2}+\epsilon_{2}, \delta_{2}, \lambda_{2}, \gamma_{2}\right]=$ $\left[r_{1}-r_{2}, \epsilon, \delta, \lambda, \gamma\right]=r_{1}-r_{2}$

3) $\overline{r_{1}} \times \overline{r_{2}}=\left[\widetilde{r_{1}}\right]_{\varepsilon} \times\left[\widetilde{r_{2}}\right]_{\varepsilon}=\left[\widetilde{r_{1} r_{2}}\right]_{\varepsilon}=\overline{r_{1} r_{2}}$ where $\widetilde{r_{1}} \times \widetilde{r_{2}}=\left[r_{1}, \epsilon_{1}, \delta_{1}, \lambda_{1}, \gamma_{1}\right] \times\left[r_{2}+\epsilon_{2}, \delta_{2}, \lambda_{2}, \gamma_{2}\right]=$ $\left[r_{1} r_{2}, \epsilon, \delta, \lambda, \gamma\right]=\widetilde{r_{1} r_{2}}$

4) $\overline{\overline{r_{1}}}=\left[\widetilde{r_{1}}\right]_{\varepsilon} /\left[\widetilde{r_{2}}\right]_{\varepsilon}=\left[\left(\frac{\widetilde{r_{1}}}{r_{2}}\right)\right]_{\varepsilon}=\overline{\left(\frac{r_{1}}{r_{2}}\right)}$ for $r_{2} \neq 0$

$$
\text { where } \frac{\widetilde{1_{1}}}{\widetilde{r_{2}}}=\frac{\left[r_{1}, \epsilon_{1}, \delta_{1}, \lambda_{1}, \gamma_{1}\right]}{\left[r_{2}, \epsilon_{2}, \delta_{2}, \lambda_{2}, \gamma_{2}\right]}=\left[\frac{r_{1}}{r_{2}}, \epsilon, \delta, \lambda, \gamma\right]=\left(\frac{\widetilde{r_{1}}}{r_{2}}\right)
$$

Thus for $r_{1}, r_{2} \in R$, we define the algebraic operations of addition and multiplication on $R_{\varepsilon}$ as follows: 


$$
\begin{gathered}
\overline{r_{1}}+\overline{r_{2}}=\left[\widetilde{r_{1}}\right]_{\varepsilon}+\left[\widetilde{r_{2}}\right]_{\varepsilon}=\left[\widetilde{r_{1}+r_{2}}\right]_{\varepsilon}=\overline{r_{1}+r_{2}} \\
\overline{r_{1}} \times \overline{r_{2}}=\left[\widetilde{r_{1}}\right]_{\varepsilon} \times\left[\widetilde{r_{2}}\right]_{\varepsilon}=\left[\widetilde{r_{1} r_{2}}\right]_{\varepsilon}=\overline{r_{1} r_{2}}
\end{gathered}
$$

Hence form the above results, we conclude that there exists an algebraic structure on $R_{\varepsilon}$.

\subsubsection{Theorem}

$R_{\varepsilon}=\left\{\bar{r}=[\widetilde{r}]_{\varepsilon} / r \in R\right\}$ is a field under fuzzy addition and fuzzy multiplication defined by

$$
\begin{gathered}
\overline{r_{1}}+\overline{r_{2}}=\left[\widetilde{r_{1}}\right]_{\varepsilon}+\left[\widetilde{r_{2}}\right]_{\varepsilon}=\left[\widetilde{r_{1}+r_{2}}\right]_{\varepsilon}=\overline{r_{1}+r_{2}} \\
\overline{r_{1}} \times \overline{r_{2}}=\left[\widetilde{r_{1}}\right]_{\varepsilon} \times\left[\widetilde{r_{2}}\right]_{\varepsilon}=\left[\widetilde{r_{1} r_{2}}\right]_{\varepsilon}=\overline{r_{1} r_{2}}
\end{gathered}
$$

\subsubsection{Theorem}

For any fuzzy $[\widetilde{r}]_{\epsilon} \in R_{\varepsilon}$ and any $n \in \mathrm{N}$, we have

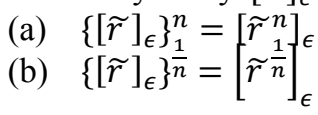

Proof: (a) Let $[\widetilde{r}]_{\epsilon} \in R_{\varepsilon}$ and $\left\{[\widetilde{r}]_{\epsilon}\right\}^{n}$ is defined as

$$
\begin{gathered}
\left\{[\widetilde{r}]_{\epsilon}\right\}^{n}=[\widetilde{r}]_{\epsilon} \times[\widetilde{r}]_{\epsilon} \times[\widetilde{r}]_{\epsilon} \times \ldots \times[\widetilde{r}]_{\epsilon}(n-\text { times }) \\
=[\widetilde{r} \times \widetilde{r} \times \ldots \times \widetilde{r}]_{\epsilon} \\
=\left[\widetilde{r}^{n}\right]_{\epsilon} \\
\text { (b) } \operatorname{Let} \widetilde{r}^{\frac{1}{n}}=\widetilde{p} \\
\widetilde{r}=\widetilde{p}^{n} \\
=[\widetilde{p}]_{\epsilon} \times[\widetilde{p}]_{\epsilon} \times[\widetilde{p}]_{\epsilon} \times \ldots \times[\widetilde{p}]_{\epsilon}(n-\text { times }) \\
=[\widetilde{p}]_{\epsilon}^{n} \\
\left\{[\widetilde{r}]_{\epsilon}\right\}^{\frac{1}{n}}=[\widetilde{p}]_{\epsilon}=\left[\widetilde{r}^{\frac{1}{n}}\right]_{\epsilon}
\end{gathered}
$$

\section{Solution of Algebraic Equations in a Fuzzy Environment}

Let us consider a fuzzy differential equation and its generalized form is given as

$$
\overline{a_{0}} \bar{x}^{n}+\overline{a_{1}} \bar{x}^{n-1}+\cdots \cdots+\overline{a_{n}}=\overline{0} ; n \in \mathrm{N}
$$

where $\overline{a_{0}}, \overline{a_{1}}, \ldots \overline{a_{n}}$ and $\overline{0}$ are fuzzy numbers and $\bar{x}$ is fuzzy variable in $R_{\varepsilon}$.

In this, we will illustrate the solution of fuzzy linear equation $\bar{a}+\bar{x}=\bar{b}$, where $\bar{a}=\overline{1}, \bar{b}=\overline{3}$ and $\bar{x}$ is the fuzzy variable.

Thus, $\overline{1}+\bar{x}=\overline{3}$

On adding $\overline{-1}$ both sides, we get

$$
\begin{gathered}
\Rightarrow \overline{-1}+(\overline{1}+\bar{x})=\overline{-1}+\overline{3} \\
\Rightarrow \quad(\overline{-1}+\overline{1})+\bar{x}=(\overline{-1}+\overline{3}) \\
\Rightarrow \quad \overline{0}+\bar{x}=\overline{(-1+3)} \\
\Rightarrow \quad \bar{x}=\overline{2} .
\end{gathered}
$$

Considering the solution of fuzzy equation in a quadratic form, we have

$$
\bar{x}^{2}-\overline{5} \bar{x}+\overline{2}=\overline{0}
$$

By comparing the above quadratic fuzzy equation from $\bar{a} \bar{x}^{2}+\bar{b} \bar{x}+\bar{c}=\overline{0}$, we have $\bar{a}=\overline{1}, \bar{b}=\overline{-5}$ and $\bar{c}=\overline{2}$.

Now, we know the discriminant is defined as

$$
\begin{gathered}
\Delta=\bar{b}^{2}-\overline{4} \bar{a} \bar{c} \\
=\overline{25}-\overline{4} \times \overline{1} \times \overline{2} \\
=\overline{25}-\overline{8} \\
=\overline{17}
\end{gathered}
$$

Thus, $\sqrt{\Delta}=\sqrt{\overline{17}}= \pm \overline{4.12}$ and the solutions are given by

$$
\begin{gathered}
\overline{x_{1}}=\frac{\overline{-b}+\sqrt{\Delta}}{\overline{2} \bar{a}} \text { and } \overline{x_{2}}=\frac{\overline{-b}-\sqrt{\Delta}}{\overline{2} \bar{a}} \\
=\frac{\overline{5}+\overline{4.12}}{\overline{2} \overline{1}}=\frac{\overline{5}-\overline{4.12}}{\overline{2} \overline{1}} \\
=\overline{4.56}=\overline{0.44}
\end{gathered}
$$

\section{Conclusions}

In this paper, we have introduced a new definition of fuzzy numbers and have developed their arithmetic and algebraic structures. Also, in this paper we have illustrated solution of fuzzy linear and quadratic equations which can be extended to higher order in future. In many real life situations, we come across problems having more than one linear equations or equations having no exact solution in which it is not possible to find solutions using analytical methods. The developed theory can be applied to solve such problems. There are many engineering and science practical application problems where these method of algebraic equation can be used.

\section{REFERENCES}

[1] Zadeh, L. A., Fuzzy Sets, Information and Control, 8(3), 338353, 1965.

[2] Atanassov, K.T., Intuitionistic fuzzy sets, Fuzzy Sets and Systems, 20(1), 87-96, 1986.

[3] Dubois, D., Prade, H., Operations on Fuzzy Numbers, International Journal of Systems Science, 9(6), 613-626, 1978.

[4] Makwana, V. C., Soni, V. P., Bhatt, P. J., Solution of Fuzzy Algebraic Equations For New Fuzzy Number, IJSRSET, 2(4), 124-130, 2016.

[5] Piasecki, K.; Łyczkowska-Hanćkowiak, A. Oriented Fuzzy Numbers vs. Fuzzy Numbers. Mathematics, 9, 523, 2021.

[6] Pathinathan, T., Ponnivalavan, K., Diamond Fuzzy Number, Journal of Fuzzy Set Valued Analysis, 2015 (1), 36-44, 2015.

[7] Buckley, J. J., Solving fuzzy equations, Fuzzy Sets and System, 50(1), 1-14, 1992. 
[8] Dubois, D., Prade, H., Fuzzy Set Theoretic Differences and Inclusions and their use in the analysis of Fuzzy Equations, Control Cybern (Warshaw), 13, 129-146, 1984.

[9] Sanchez, E., Solution of fuzzy equations with Extended Operations, Fuzzy Sets and System, 12, 273-248, 1984.

[10] Kawasaki, J., On solution of fuzzy equation, Control and Cybern, 26, 653-658, 1997.

[11] Chou, Chien-Chang, The Square Roots of Triangular Fuzzy Number, ICIC Express Letters, 3(2), 207-212, 2009.

[12] Goetschel, R., Voxman, W. Elementary fuzzy calculus, Fuzzy Sets Syst., 18, 31-43, 1986.

[13] Verma, R., Merigó, J.M., Sahni, M., Pythagorean fuzzy graphs: some results, arXiv preprint arXiv:1806.06721, 2018.
[14] Sahni, M. Sahni, R., Verma, R. Mandaliya, A., Shah, D., Generalized trapezoidal intuitionistic fuzzy number for finding radial displacement of a solid disk, WSEAS Transactions on Mathematics, 18, 105-111, 2019.

[15] Sahni, M., Parikh, M., Sahni, R., Sumudu transform for solving ordinary differential equation in a fuzzy environment, Journal of Interdisciplinary Mathematics, 24(6), 1565-1577, 2021.

[16] Esa, R. I., Ibraheem, R.H., Jameel, A. F., Numerical Treatment for solving Fuzzy Volterra Integral Equation by Sixth Order Runge-Kutta Method, Mathematics and Statistics, 9(3), 350-356, 2021. DOI: 10.13189/ms.2021.090317.

[17] Delgado, M., Vila, M.A., Voxman, W., On a canonical representation of fuzzy numbers. Fuzzy Sets Syst., 93, 125135, 1998. 